\title{
Bolus-Infusion Delays of Alteplase during Thrombolysis in Acute Ischaemic Stroke and Functional Outcome at 3 Months
}

\author{
Paul Acheampong, ${ }^{1}$ Margaret T. May, ${ }^{2}$ Gary A. Ford, ${ }^{1,3}$ and Anand K. Dixit ${ }^{1}$ \\ ${ }^{1}$ Acute Stroke Service, Royal Victoria Infirmary, Newcastle upon Tyne NE1 4LP, UK \\ ${ }^{2}$ School of Social and Community Medicine, University of Bristol, Bristol BS8 2PS, UK \\ ${ }^{3}$ Institute for Ageing and Health, Newcastle University, Newcastle upon Tyne NE2 4HH, UK \\ Correspondence should be addressed to Anand K. Dixit; anand.dixit@nuth.nhs.uk
}

Received 6 February 2014; Revised 7 April 2014; Accepted 7 April 2014; Published 30 April 2014

Academic Editor: David S. Liebeskind

Copyright (C) 2014 Paul Acheampong et al. This is an open access article distributed under the Creative Commons Attribution License, which permits unrestricted use, distribution, and reproduction in any medium, provided the original work is properly cited.

\begin{abstract}
Background. The efficacy of alteplase in acute ischaemic stroke (AIS) is highly time dependent. Hence, alteplase is administered as soon as possible with a bolus followed by an infusion. Delays between bolus and infusion may occur, but the extent of these delays and the impact on outcome are unclear. Aims. We investigated the extent of bolus-infusion delays and the relationship between delays and stroke outcome. Method. We reviewed medical records of 276 patients who received alteplase for AIS at our centre between April, 2008, and June, 2013. Complete demographic and clinical data including 3-month modified Rankin Score (mRS) from 229 patients were analysed comparing delays of $0-8$ and $>8$ minutes. Results. Overall mean (SD) bolus-infusion delay was 9 (7) minutes. Baseline characteristics were similar apart from more severe strokes in delays $>8$ minutes. Three-month outcomes were not significantly different although delays $>8$ minutes had lower functional independence rate (mRS $0-1: 23.1 \%$ versus $28.1 \%$; adjusted OR $1.2(95 \%$ CI 0.6 to $2.4, P=0.68)$ ) and higher mortality rate (18\% versus $11 \%$, OR $1.0,95 \%$ CI 0.6 to $1.7, P=0.95)$. Conclusions. In this single centre series, bolus-infusion delays of alteplase in AIS were common and no effect of bolus-infusion delays on independence and mortality was found.
\end{abstract}

\section{Introduction}

Alteplase is licensed for use in acute ischaemic stroke (AIS). Its efficacy in improving stroke outcome depends on onset to treatment time (OTT) [1]. Alteplase has a short plasma half-life of about 4 minutes [2] and is administered at a dose of $0.9 \mathrm{mg} / \mathrm{kg}$ body weight with $10 \%$ of the total dose given as a bolus over 1-2 minutes and the remainder infused over an hour. This regimen has been demonstrated to achieve functional improvement with a low risk of intracranial haemorrhage [3].

None of the trials of alteplase in AIS reported the extent of bolus-infusion delays. Indeed, there are very limited data on the pharmacokinetic profile of alteplase as used in AIS $[4,5]$. Considering the short half-life of alteplase, significant bolusinfusion delays may result in delayed or suboptimum steady state kinetics which could adversely influence outcome.
We investigated the extent of bolus-infusion delays of alteplase in AIS in a single centre and its relationship with functional outcome at 3 months.

\section{Materials and Methods}

We retrospectively reviewed medical records of patients treated with alteplase within $4.5 \mathrm{~h}$ after AIS at Newcastle upon Tyne Hospitals Trust from April 2008 to June 2013. Data on baseline demographics and relevant clinical parameters, functional outcome based on modified Rankin Scale (mRS) score at 3 months, and time lapse between bolus and infusion doses of alteplase were retrieved.

Bolus-infusion delays have been reported per typical halflives (4 minutes) and further categorized as $0-8$ and $>8$ minutes for inference analysis based on the likelihood of significant decline in plasma concentration of alteplase after 
a bolus-infusion delay of 2 half-lives [5]. Demographic and clinical data have been reported as descriptive summaries with inference statistics using independent $t$-test and chisquare $\left(\chi^{2}\right)$ test.

We analysed the relationship between independent functional outcome (mRS $0-1$ and $0-2$ ) at 3 months and bolusinfusion delays of $0-8$ and $>8$ minutes using multivariable logistic regression (MLR) adjusting for age, sex, blood pressure, atrial fibrillation and hyperlipidaemia status, antiplatelet use, prestroke mRS, Oxfordshire Community Stroke Project classification, prethrombolysis NIHSS, and OTT. Patients who received at least two-thirds of the total recommended intravenous dose of alteplase without intra-arterial or thrombectomy interventions were included in the analysis based on pharmacokinetic models which predict attainment of $\geq 90 \%$ of the potential peak plasma concentration from full dose alteplase assuming a bolus injection time of 1 minute and bolus-infusion delay of 5-15 minutes [5]. IBM SPSS version 20 (New York, USA) and Stata statistical software version 12 (College Station, Texas, USA) were used for the analysis.

\section{Results}

Medical records of 276 patients with AIS treated with intravenous thrombolysis were reviewed. A total of 229 patients were included in the analysis after the exclusion of 47 patients with incomplete data involving admission glucose, infusion start time and/or mRS at 3 months $(n=24)$, endovascular treatment including intra-arterial thrombolysis and thrombectomy $(n=17)$, and administration of less than two-thirds of total dose of alteplase mainly due to early termination of alteplase infusion $(n=6)$. Demographic and clinical characteristics of excluded patients were similar to those included in the analysis.

Baseline characteristics were similar between the groups with bolus-infusion delays of $0-8$ and $>8$ minutes except for the latter group that had more severe strokes as determined by prethrombolysis NIHSS (Table 1). Bolus-infusion delays of $0-4,5-8,9-12$, and $>12$ minutes occurred in $20 \%, 33 \%, 25 \%$, and $22 \%$ of patients, respectively, with an overall mean (SD) delay of 9 (7) minutes.

Unadjusted functional independence at 3 months was similar among the two groups (mRS 0-2: $50 \%$ versus $50 \%$, continuity correction $\chi^{2}$ test $=0$, unadjusted OR $1.0,95 \% \mathrm{CI}$ 0.6 to 1.7 , and $P=0.95$. mRS $0-1: 28.1 \%$ versus $23.1 \%$ for delays of $0-8$ and $>8$ minutes, resp., continuity correction $\chi^{2}$ test $=$ 0.495 , unadjusted OR $0.8,95 \%$ CI 0.4 to 1.4 , and $P=0.39$ ). Adjusted OR for an independent functional outcome for delays $>8$ minutes was marginally significant at 2.1 (95\% CI 1.0 to $4.1, P=0.04$ ) based on mRS 0-2 but insignificant using mRS $0-1$ at 1.2 (95\% CI 0.6 to $2.4, P=0.68$ ). As expected, independent functional outcome at 3 months was adversely affected by higher prethrombolysis NIHSS score. Compared with NIHSS 0-9, unadjusted OR for good functional outcome were 0.28 (95\% CI 0.14 to $0.56, P<0.001$ ) and 0.07 (95\% CI 0.02 to $0.25, P<0.001)$ for NIHSS $10-19$ and $>19$, and adjusted OR were 0.46 (95\% CI 0.18 to $1.19, P=0.11)$ and 0.10 (95\% CI 0.02 to $0.42, P=0.002$ ), respectively. The mortality rate at 3 months was nonsignificantly higher in the group with

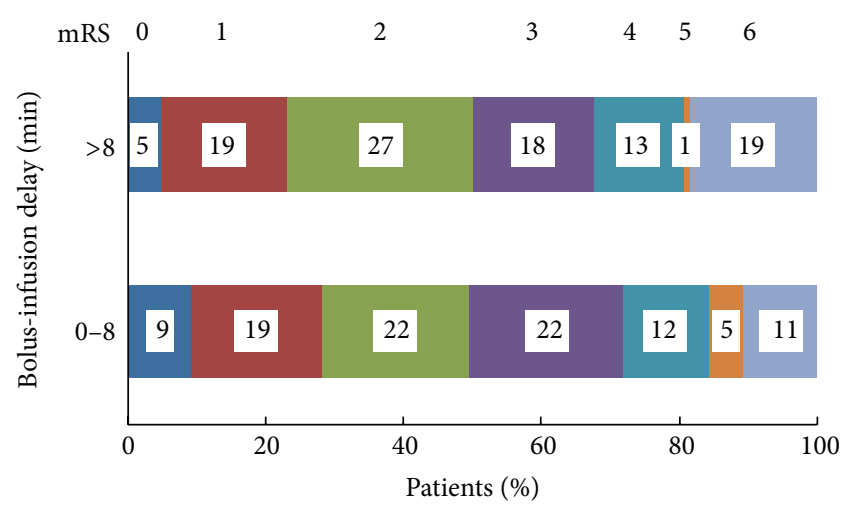

FIGURE 1: Bolus-infusion delays during treatment with intravenous alteplase within $4.5 \mathrm{~h}$ and outcome at 3 months after stroke. mRSmodified Rankin Scale.

delays $>8$ minutes $(11 \%$ versus $18 \%$, difference $7.8, \mathrm{CI}-2.2$ to 17.8 , continuity correction $\chi^{2}$ test $=2.2$, and $P=0.138$ ) (Figure 1).

\section{Discussion}

In this single centre study, we observed bolus-infusion delays of $>8$ minutes in $47 \%$ of AIS patients treated with intravenous alteplase. Compared to those with delays $>8$ minutes, bolus- infusion delays of $\leq 8$ minutes were associated with nonsignificantly higher independent functional outcome and lower mortality rates after 3 months. Our findings are similar to those reported in abstract form in a smaller series of 120 patients where no difference in functional outcome or recanalisation was found [6].

In order to minimise OTT and optimise outcome, stroke pathways have evolved to allow the administration of bolus doses of alteplase as quickly as possible, sometimes in the CT or MRI scanner. The infusion may then be started after patients have been transferred to the acute stroke ward, emergency department, or clinical treatment areas resulting in delays between the administration of the bolus dose and commencement of the infusion. We observed longer bolusinfusion delays in patients with more severe strokes. This might be due to a longer time taken to transfer the patients from the CT table to the trolley which in our institution takes place before the infusion is commenced.

In healthy cohort and myocardial infarction studies using intravenous alteplase, the administration of a bolus dose immediately before commencement of infusion led to the attainment of steady state concentration in about 4 minutes [7] compared to about 20 minutes for infusion only dosing [2]. With a mean bolus-infusion delay of 9 minutes (approximately 2 half-lives) in our cohort, peak plasma concentrations after the administration of the bolus dose of alteplase would have significantly dropped by about $75 \%$ at the time of initiation of the infusion. Moreover, the infusion regimen delivers $1.5 \%$ of the total dose of alteplase per minute compared to $10 \%$ for the bolus if given over a minute. In cases of significant bolus-infusion delays, therefore, the contribution of the bolus injection towards 
TABLE 1: Demographic and clinical characteristics of patients. Data presented as mean [SD] for continuous variables and proportions (\%) for categorical variables unless otherwise stated.

\begin{tabular}{|c|c|c|}
\hline \multirow{2}{*}{ Characteristics } & \multicolumn{2}{|c|}{ Bolus-infusion delays } \\
\hline & $\begin{array}{c}0-8 \text { minutes } \\
(n=121)\end{array}$ & $\begin{array}{l}>8 \text { minutes } \\
(n=108)\end{array}$ \\
\hline Male sex & 50 & 56 \\
\hline Mean age (years) & $73[11]$ & $72[12]$ \\
\hline Mean weight (kg) & $72[14]$ & $76[16]$ \\
\hline Mean systolic blood pressure (mmHg) & $144[22]$ & $142[22]$ \\
\hline Mean diastolic blood pressure ( $\mathrm{mmHg}$ ) & $78[15]$ & $76[15]$ \\
\hline Mean admission blood glucose $(\mathrm{mmol} / \mathrm{L})$ & $7[2]$ & $7[3]$ \\
\hline Hypertension & 71 & 59 \\
\hline Diabetes mellitus & 13 & 12 \\
\hline Atrial fibrillation & 37 & 45 \\
\hline Congestive cardiac failure & 8 & 4 \\
\hline Hyperlipidaemia & 45 & 45 \\
\hline TIA/amaurosis fugax & 10 & 6 \\
\hline Previous stroke & 14 & 12 \\
\hline Peripheral vascular disease & 6 & 5 \\
\hline Myocardial infarction or ischaemic heart disease & 19 & 30 \\
\hline Current smoker & 15 & 18 \\
\hline Ex-smoker & 26 & 22 \\
\hline Antiplatelet use & 53 & 60 \\
\hline Pre-stroke mRS, median (IQR) & $1(0-2)$ & $1(0-2)$ \\
\hline \multicolumn{3}{|c|}{ Mean onset to treatment time (OTT) in minutes; proportions within groups } \\
\hline $0-90$ & $79[11] ; 11 \%$ & $74[16] ; 18 \%$ \\
\hline $91-180$ & $132[25] ; 63 \%$ & $132[26] ; 64 \%$ \\
\hline $181-270$ & $212[19] ; 26 \%$ & $209[22] ; 18 \%$ \\
\hline $0-270$ & $147[47]$ & $136[47]$ \\
\hline \multicolumn{3}{|l|}{ Oxfordshire Community Stroke Project } \\
\hline Total anterior circulatory stroke & 41 & 56 \\
\hline Partial anterior circulatory stroke & 45 & 35 \\
\hline Lacunar stroke & 12 & 6 \\
\hline Posterior circulatory stroke & 2 & 4 \\
\hline \multicolumn{3}{|c|}{ Pre-thrombolysis neurological severity per NIHSS score } \\
\hline Median (IQR)* & $10[7-17]$ & $14.5[9-21]$ \\
\hline \multicolumn{3}{|l|}{ Proportions within NIHSS categories $(\%)^{* *}$} \\
\hline $0-9$ & 49 & 30 \\
\hline $10-19$ & 35 & 37 \\
\hline$>19$ & 16 & 33 \\
\hline
\end{tabular}

NIHSS-National Institutes of Health Stroke Scale; TIA-Transient Ischaemic Attack; mRS-modified Rankin Score. No significant differences between infusion delays of $0-8$ and $>8$ minutes except for * (Mann Whitney $\mathrm{U}$ test, $P=0.016$ ) and ${ }^{* *}$ (Pearson's $\chi 2$ test $=13.1, P=0.001$ ).

steady state concentration could wane resulting in a delayed or suboptimum steady state concentration with potential knock-on effects on achieving optimum fibrinolysis. This is illustrated by delays in reaching initial peak concentrations of approximately 20 and 30 minutes in studies simulating bolusinfusion delays of 5 and 15 minutes, respectively [5].

Intuitively, a shorter bolus-infusion delay was postulated to be associated with improved outcome based on the attainment of near optimum plasma alteplase concentrations. The largely neutral three-month outcome measures may reflect the higher antiplatelet use and shorter mean OTT, which are associated with better outcome $[1,3]$ in the group with delays $>8$ minute albeit with a higher prethrombolysis median NIHSS (Table 1). It is possible that the effect of alteplase on the fibrinolytic system in AIS is not only defined by the optimum steady state concentrations envisaged from the immediate commencement of the infusion after the bolus. Other factors such as the speed of delivery of alteplase to, and saturation 
of, the plasminogen catalytic sites within the thrombus as evident in the effect of OTT on outcome after AIS, may also influence this [1]. This is consistent with the rationale for the departure from a complete reliance on infusions as employed in earlier trials [8].

Our study has a number of limitations. The data were retrospectively retrieved from a single centre with over 15year experience of administering alteplase for AIS and it is possible that delays may vary in other centres.

\section{Conclusions}

Our study demonstrates that bolus-infusion delays are likely to be common. Delays of $>8$ minutes were not associated with significantly worse outcomes although the functional independence rate was lower and death rate was higher in this group. The series is however small to conclusively define the association between bolus-infusion delays and outcomes, as such larger prospective studies will be useful.

\section{Conflict of Interests}

The authors declare that there is no conflict of interests.

\section{References}

[1] K. R. Lees, E. Bluhmki, R. von Kummer et al., "Time to treatment with intravenous alteplase and outcome in stroke: an updated pooled analysis of ECASS, ATLANTIS, NINDS, and EPITHET trials," The Lancet, vol. 375, no. 9727, pp. 1695-1703, 2010.

[2] P. Tanswell, E. Seifried, P. C. A. F. Su, W. Feuerer, and D. C. Rijken, "Pharmacokinetics and systemic effects of tissue-type plasminogen activator in normal subjects," Clinical Pharmacology and Therapeutics, vol. 46, no. 2, pp. 155-162, 1989.

[3] The National Institute of Neurological Disorders and Stroke rtPA Stroke Study Group, "Tissue plasminogen activator for acute ischemic stroke," The New England Journal of Medicine, vol. 333, pp. 1581-1571, 1995.

[4] P. Acheampong and G. A. Ford, "Pharmacokinetics of alteplase in the treatment of ischaemic stroke," Expert Opinion on Drug Metabolism and Toxicology, vol. 8, no. 2, pp. 271-281, 2012.

[5] C. Smith, Y. Al-Nuaimi, J. Wainwright et al., "The influence of bolus to infusion delays on plasma Tissue Plasminogen Activator levels," International Journal of Stroke, 2012.

[6] X. Huang and K. W. Muir, "Does alteplase bolus infusion delay affect outcome?" International Journal of Stroke, vol. 7S2, article 13, 2012.

[7] E. Seifried, P. Tanswell, D. Ellbruck, W. Haerer, and A. Schmidt, "Pharmacokinetics and haemostatic status during consecutive infusions of recombinant tissue-type plasminogen activator in patients with acute myocardial infarction," Thrombosis and Haemostasis, vol. 61, no. 3, pp. 497-501, 1989.

[8] E. C. Haley Jr., D. E. Levy, T. G. Brott et al., "Urgent therapy for stroke: part II. Pilot study of tissue plasminogen activator administered 91-180 minutes from onset," Stroke, vol. 23, no. 5, pp. 641-645, 1992. 


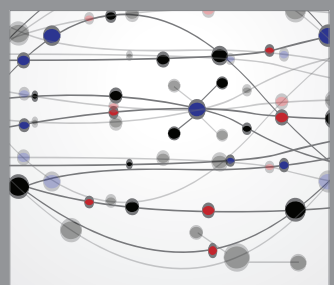

The Scientific World Journal
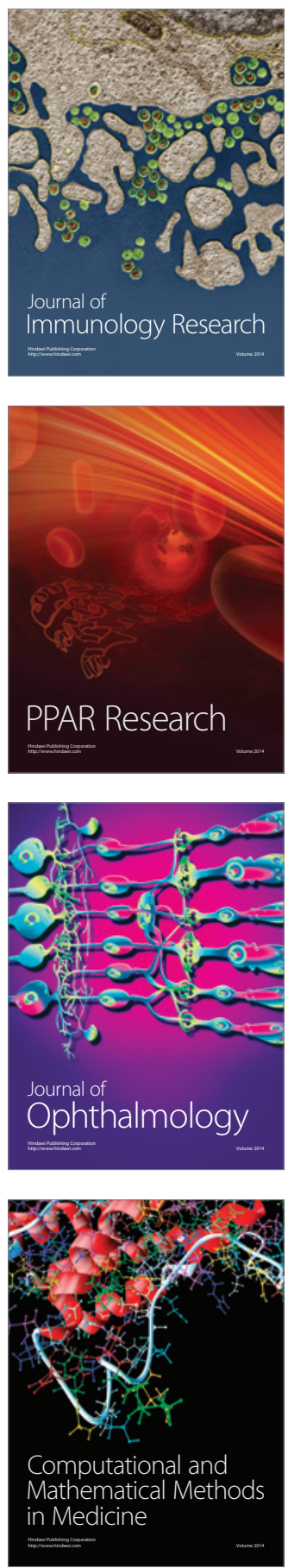

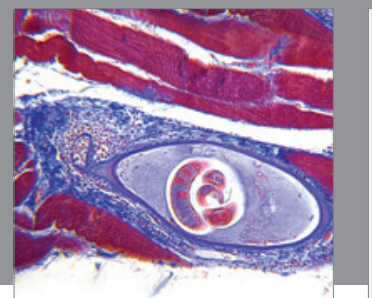

Gastroenterology

Research and Practice
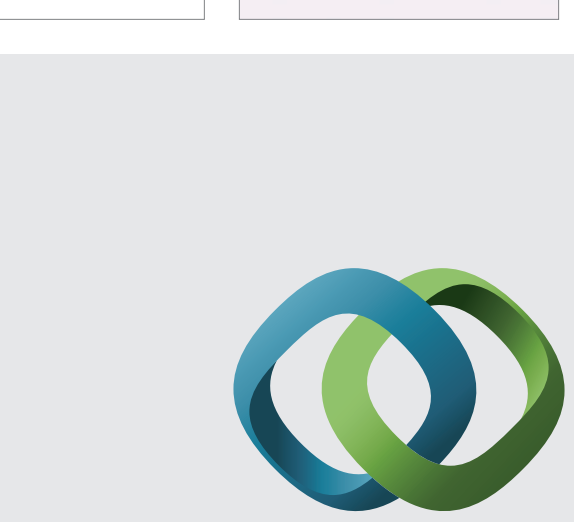

\section{Hindawi}

Submit your manuscripts at

http://www.hindawi.com
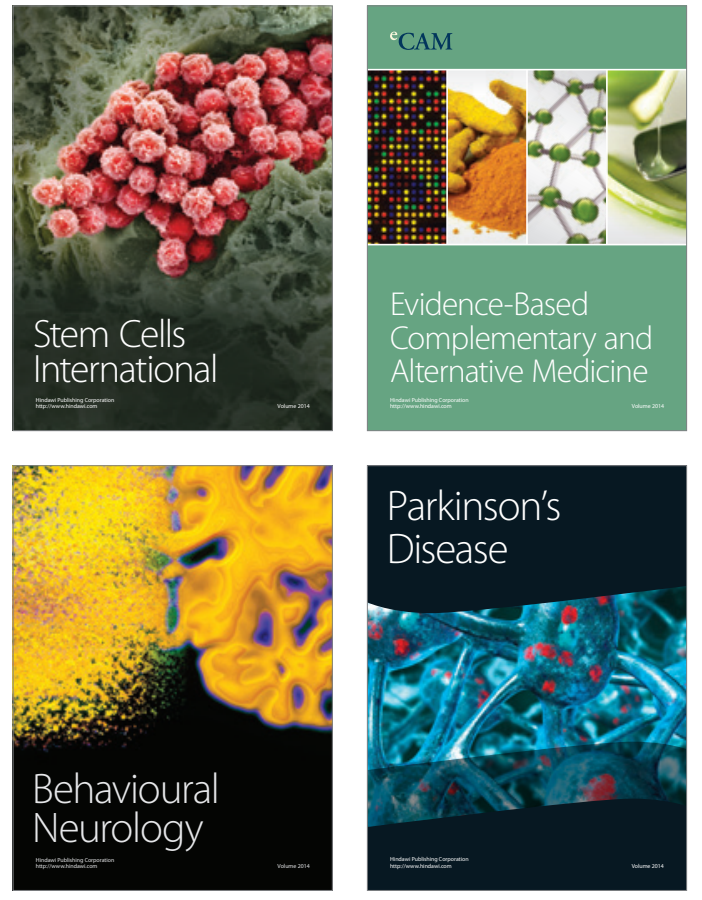
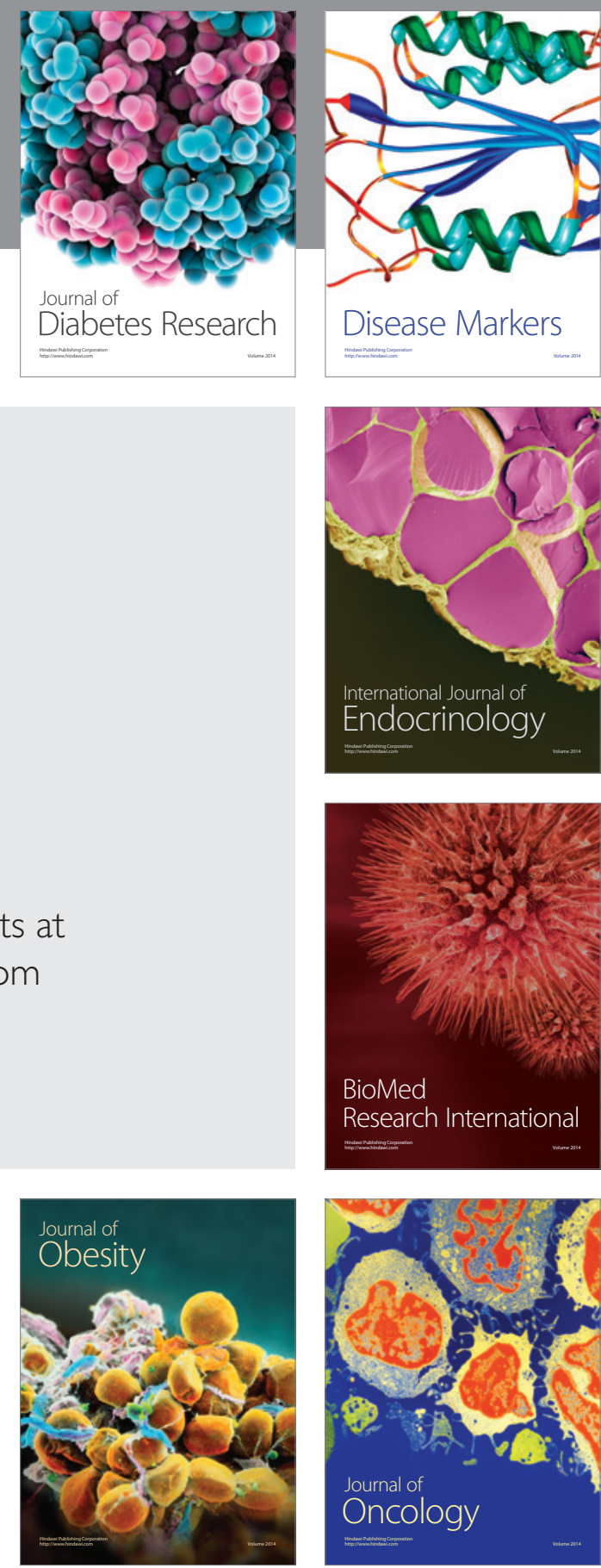

Disease Markers
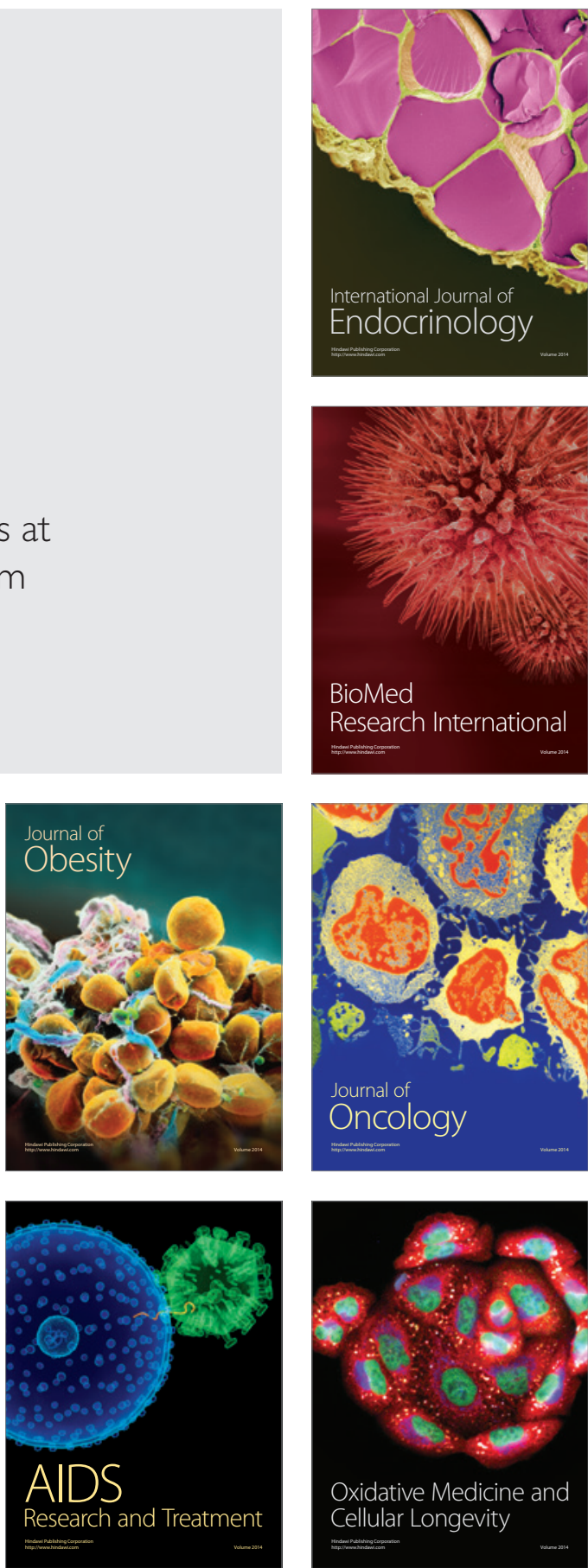